SPECIAL ISSUE

\title{
The Mobile Teleophthalmology Unit in Rural and Underserved Areas of South India
}

\author{
Sheila John'*, Lavanya Allimuthu', Ranjitha Kannan', Ramesh BabuSekar', Martin Manoj \\ Mathiyazahan', Padmavathy Appasamy', Sangeetha Srinivasan² \\ 'Department of Teleophthalmology, Sankara Nethralaya, Chennai, India; ${ }^{2}$ Vision Research Foundation, Chennai, India
}

Abstract

Objective: Our objective was to provide an eye care service to rural and underserved areas in Chennai, Kanchipuram, and Thiruvallur districts of Tamil Nadu, South India.

Design: We conducted eye camps to provide ophthalmic services to the underserved and rural areas, where people cannot afford to go to a hospital due to lack of accessibility, lack of awareness, or financial constraints.

Setting: The study was conducted in rural and underserved areas of Thiruvallur, Chennai, and Kanchipuram districts from January 2015 to December 2019.

Participants: Patients $(N=1,05,827)$ underwent comprehensive eye examination in eye camps with the stateof-art ophthalmic equipment.

Main outcome measures: To report on the number of patients examined, number of eye disorders screened, and different types of ocular pathology screened, all clinical findings were recorded and all ocular images were uploaded in the electronic medical records. All patients with ocular diseases underwent teleconsultation with an ophthalmologist at the base hospital with internet connectivity. Video conferencing and teleconsultation were feasible only in areas with good internet connectivity.

Results: Over the 5-year study period, 1,05,827 patients underwent eye evaluation at 1,061 eye camps. Among these, 48,354 (45.7\%) patients were males, 57,473 (54.3\%) patients were females, 15,515 patients were emmetropes. The most common cause of avoidable blindness was uncorrected refractive error detected in 66,137 eyes, referable cataract was seen in 13,536 eyes, 2,491 eyes were identified to have retinal diseases, and there were 789 patients with only diabetic retinopathy, thus totaling to 3,280 comprising of all retinal disease. 2424 patients received teleconsultations. For further investigations and treatment, which were provided free of cost, patients were referred to the base hospital in Chennai. There were 6,309 patients who received free spectacles and an additional 31,192 patients received spectacles at a low cost; 13,536 patients had referable cataract and were referred to the base hospital for further evaluation and surgery.

Conclusions: Teleophthalmology holds great potential to overcome barriers, improve quality, access, and affordability to eye care, and has proven to be an innovative means of taking comprehensive eye care facilities to the doorsteps of rural India.

Keywords: India; electronic medical records; ocular pathology; ophthalmic services; mobile eye care; teleconsultation; telehealth; telemedicine; teleophthalmology; video conferencing

$\mathrm{T}$ eleophthalmology has been gaining acceptance worldwide as an effective eye care delivery model, especially for extending the reach of specialty eye care to medically underserved populations and rural areas where people are affected by visual impairment. The World Health Organization (WHO) in their recent report stated that at least 1 billion people worldwide are estimated to have preventable visual impairment (1). There are 7.8 million blind people in India, the second highest population of blind people in the world. About $90 \%$ of the world's visually impaired live in low-income settings of which, $82 \%$ of visual impairment can be prevented and cured. The main cause behind accessibility and affordability of the eye care service is the low-income setting. Cataract and refractive errors are still the major cause of avoidable blindness in India. We conduct eye camps in rural areas and to those who don't have access to eye care facility and provide them a comprehensive eye examination, dispense 
spectacles and prescription free of cost, and refer those that need further evaluation, treatment, or surgery to the base hospital.

In India, there is a paucity of ophthalmologist with just one ophthalmologist to 100,000 population (2). Teleophthalmology can thus help reduce the need for travel for both the patient and ophthalmologist by providing access to specialist care in remote areas. With the help of electronic medical records (EMR) and good network connectivity, it is possible to efficiently transfer information from one place to another (3).

With the aim of providing comprehensive and quality eye care to the rural population, a unique program implementing a mobile teleophthalmology unit (4) was designed at our hospital and launched in the year 2003. The scope of this venture has expanded in terms of expertise, equipment, and successful partnerships. This report provides an overview of the current process of conducting comprehensive eye camps with an emphasis on teleophthalmology consultation and review of results of this endeavor over the past 5 years. Initially, teleconsultations were done using satellite connectivity provided by (Fig. 1) Indian Space Research Organization (ISRO), and now, we use only internet connectivity.

\section{Methods}

To successfully carry out a comprehensive eye camp, one needs a good understanding of the various epidemiologic aspects of blindness in the targeted population, skilled manpower, and enormous technological support. Teleophthalmology camps involve the following activities: identifying the camp location, planning the camp, conducting eye camps, teleconsultation, providing low-cost spectacles to the rural population, referral of surgical cases to the base hospital, and conducting counseling/awareness programs and follow-up on the effect of the program.
This teleophthalmology outreach program was developed by our institution to serve people in rural areas and in areas where there is limited access to ophthalmic care. Ethics approval was provided by our Institutional Review Board.

The outreach program was conducted (Fig. 2) in Thiruvallur, Chennai, and Kanchipuram districts in the rural and underserved areas of Tamil Nadu, South India, from January 2015 to December 2019. This was a cross-sectional study that examined people across various age groups who attended the camp. There was no exclusion. Participants provided consent for eye examination.

\section{Clinical procedure}

A total of $1,05,827$ patients were examined. Every step was explained to the patient before the initiation of the procedure, after which the consent form was signed. Information such as patient name, age, sex, and marital status were registered in the system, followed by a clinical examination. Camp patients underwent a detailed history-taking, refraction using an auto-refractor, subjective refractive, retinoscopy (if required), pupillary evaluation, near point of convergence and accommodation (if required), slit lamp examination and measurement of intraocular pressure, nonmydriatric fundus photography (a single field 45 degrees centered on the macula) after dark adaption, spectacle dispensing, and counseling. All details are updated in the offline EMR, so that the patient details can be easily accessed at any time. As diagnosis of many eye diseases is image based, integrating images with clinical notes at every visit and storing them into EMR will be very useful, especially in chronic diseases such as glaucoma, diabetic retinopathy, and macular degeneration. Awareness programs on various eye diseases were conducted at the campsite for the waiting patients.

Retinal images were converted to JPEG images and transferred to the base hospital along with slit lamp

a. Mobile unit with satellite dish connectivity

b. Mobile van with internet connectivity

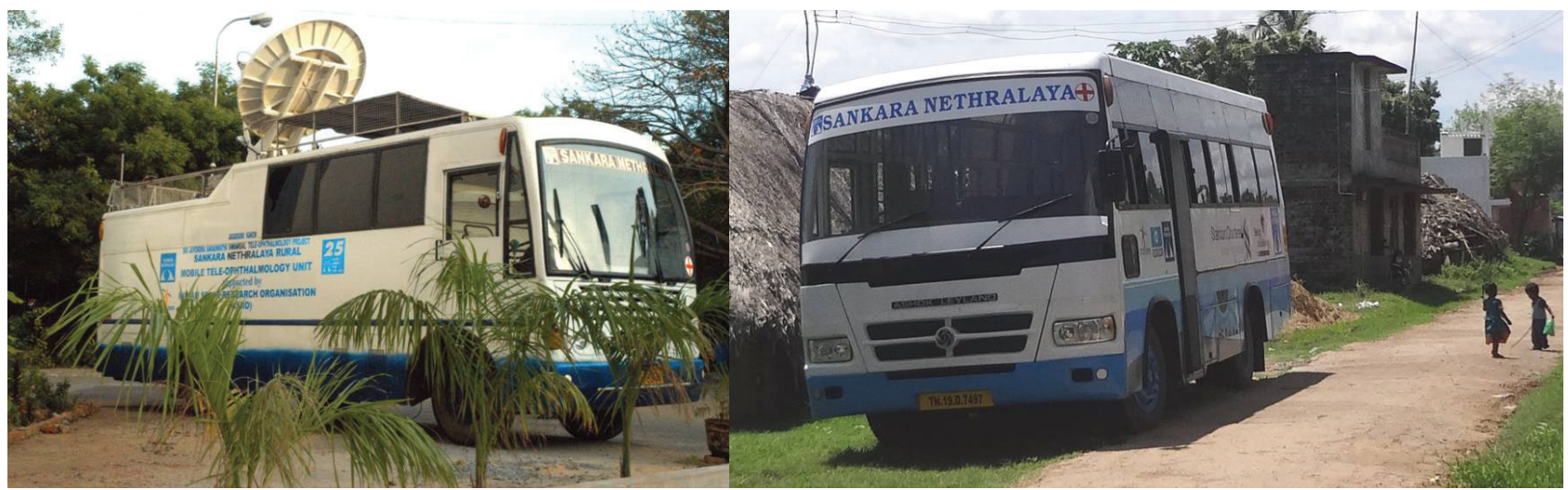

Fig. 1. Teleophthalmology mobile unit. 


\section{TAMIL NADU DISTRICT MAP}

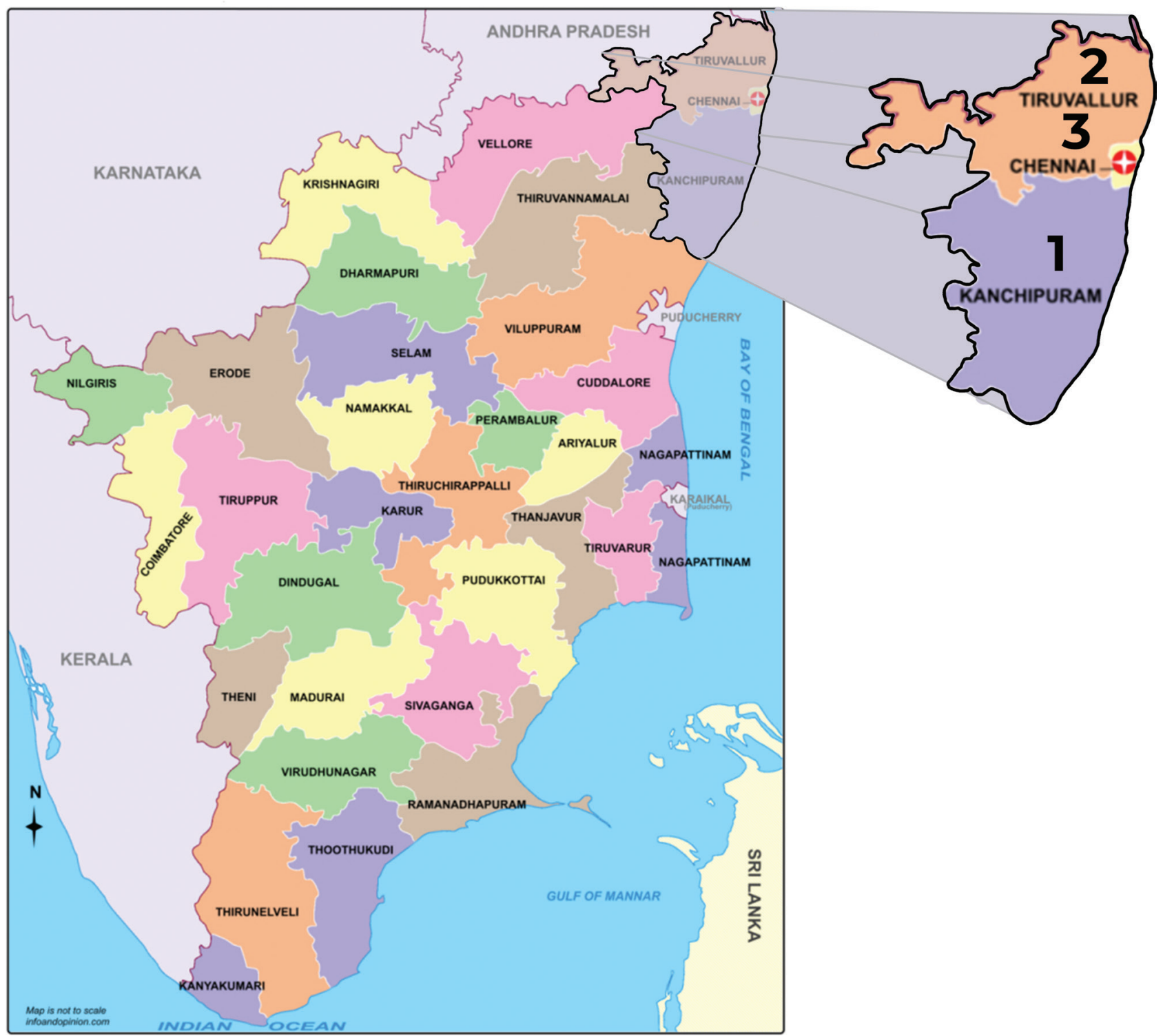

Fig. 2. Tamil Nadu district map in India.

images by a broadband connectivity and real-time video-conferencing when possible. The transmission rate ranged from $2 \mathrm{mbps}$ to $384 \mathrm{kbps}$.

Patients with issues such as unexplained vision loss, eye infection, family history of diabetes, suspected glaucoma, optic nerve problem, any history of ocular surgeries associated with visual impairment, and history of trauma were counseled by optometrists and interacted with specialist ophthalmologist at our hospital via video call teleconsultation at the camp site.

At the base hospital, an ophthalmologist evaluated the photographs and clinical data provided. Real-time interaction (Fig. 3) between the ophthalmologist, optometrist, patients and the patient attendant was facilitated via a videoconferencing system.

The examining optometrist carried out further examination when advised by the ophthalmologist. Medical treatment advised was then dispensed in the outreach facility, and patients needing further investigations, medical, and surgical treatments were transported back to the base hospital.

All our outreach camps ensure quality, especially in terms of screening vision-threatening diseases, referral services, and affordable, rapid rehabilitation of vision. 


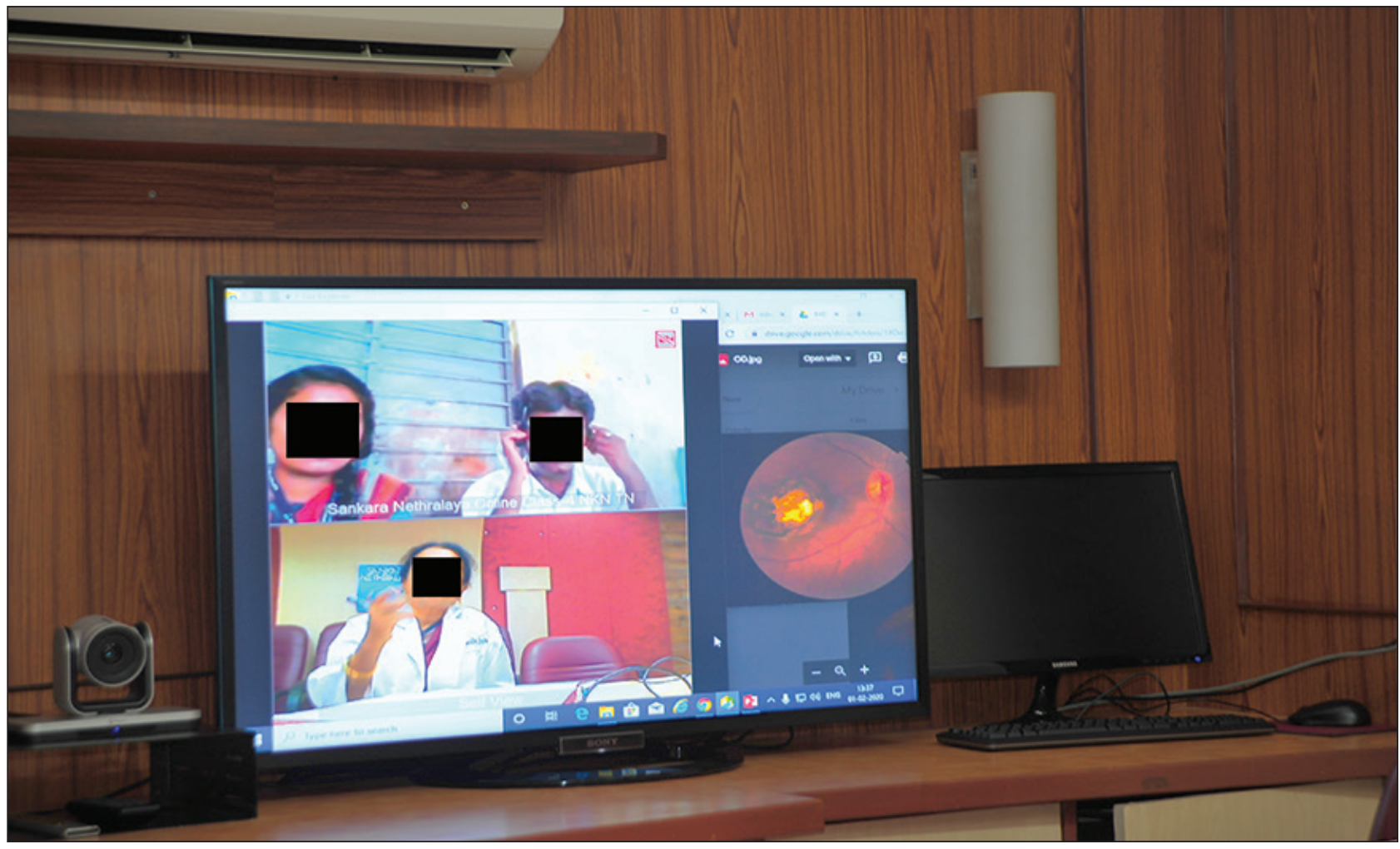

Fig. 3. Real-time interaction between the ophthalmologist, optometrist and patient.

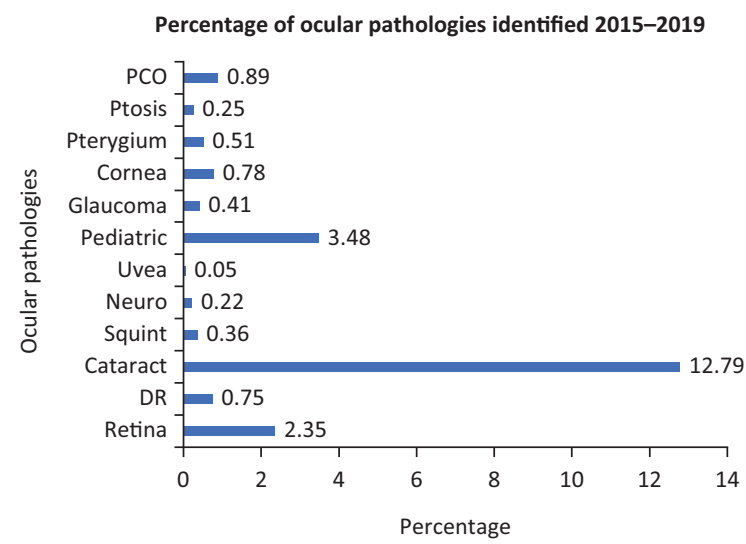

Fig. 4. Percentage of ocular pathologies identified in teleophthalmology camps: PCO, posterior capsule opacification; DR, diabetic retinopathy.

\section{Results}

The study was conducted from April 2015 to March 2019. A total $1,05,827$ patients underwent eye examination; $48,354(45.7 \%)$ patients were males, and 57,473 (54.3\%) patients were females. The ocular conditions (Fig. 4) of patients which we screened in our eye camps were as follows: 3,280 patients had retinal condition, 789 patients had only diabetic retinopathy; 13,536 patients had referable cataract, 378 patients had squint, 237 patients had neural condition, 51 patients had uveal condition, 3,687 patients had pediatric disease, 436 patients had glaucoma, 823 patients had corneal problem, 539 patients had pterygium, 267 patients had ptosis, 941 patients had posterior capsule opacification (PCO) (Fig. 4), 15,515 patients were emmetropic, 66,137 patients had refractive error $(15,525$ had myopia, 22,552 had hyperopia, 7,831 had with the rule astigmatism, 6,021 had against the rule astigmatism), and 14,208 had presbyopia (Table 1).

Referral patients who required further investigation and treatment were sent to our base hospital in the teleophthalmology department van. All investigations and surgeries for referred patients are done at the base hospital including cataract surgery and other ocular surgeries such as squint, pterygium, glaucoma, cornea, and retinal surgeries. Surgery cost, post-operative reviews, glasses appointment reviews, and glasses were provided free of cost.

\section{Discussion}

Teleophthalmology and EMR have unlocked a new frontier in screening and diagnosing of common ophthalmic diseases. Teleophthalmology can reduce the need for travel for the patient and provide access to specialist by remote means, and EMR can efficiently transfer information on diagnosis from one place to another. 
Table 1. Number of patients examined from 2015 to 2019

\begin{tabular}{|c|c|c|c|c|c|c|}
\hline Year & 2015 & 2016 & 2017 & 2018 & 2019 & Total \\
\hline Male & 8,358 & 12,057 & 9,683 & 8,819 & 9,437 & 48,354 \\
\hline Female & 8,978 & 12,117 & 12,500 & $\mathrm{II}, 804$ & 12,074 & 57,473 \\
\hline Population examined in total & 17,336 & 24,174 & 22,183 & 20,623 & 21,511 & 105,827 \\
\hline Retina & 348 & 322 & 751 & 541 & 529 & 2,491 \\
\hline Diabetic retinopathy & 236 & 157 & 263 & 81 & 52 & 789 \\
\hline Cataract & 2,105 & 2,509 & 3,985 & 2,653 & 2,284 & 13,536 \\
\hline Squint & 63 & 126 & 114 & 40 & 35 & 378 \\
\hline Neuro & 33 & 63 & 91 & 35 & 15 & 237 \\
\hline Uvea & 4 & 8 & 36 & 2 & I & 51 \\
\hline Pediatric & 246 & 475 & 292 & 2,052 & 622 & 3,687 \\
\hline Glaucoma & 47 & 51 & 149 & 97 & 92 & 436 \\
\hline Cornea & 105 & 177 & 277 & 152 & 112 & 823 \\
\hline Pterygium & 91 & 109 & 158 & 85 & 96 & 539 \\
\hline Ptosis & 22 & 52 & 122 & 39 & 32 & 267 \\
\hline Posterior capsular opacity & $14 \mid$ & 240 & 257 & 190 & 113 & 941 \\
\hline Emmetropia & 5,287 & 2,687 & 3,015 & 1,488 & 3,038 & $15,5 \mid 5$ \\
\hline Presbyopia & $\mathrm{I}, 478$ & 5,732 & 1,590 & 2,666 & 2,742 & 14,208 \\
\hline Myopia & 1,977 & 4,333 & 3,284 & 2,011 & 3,920 & 15,525 \\
\hline Hyperopia & 2,812 & 5,866 & 3,782 & 4,349 & 5,743 & 22,552 \\
\hline Astigamatism with rule & 731 & 837 & $\mathrm{I}, 862$ & 3,143 & $\mathrm{I}, 258$ & 7,831 \\
\hline Astigamatism against rule & 1,610 & 430 & 2,155 & 999 & 827 & 6,021 \\
\hline Overall refractive errors & 8,608 & 17,198 & 12,673 & 13,168 & 14,490 & 66,137 \\
\hline
\end{tabular}

Comprehensive eye camps utilizing a mobile teleophthalmology unit appear to be a very useful tool to improve eye care delivery in the developing world, especially in the rural areas. Madras Diabetic Research Foundation (MDRF), Chennai, community outreach program (5) serves a cluster of 42 villages (in and round Chunampet village) in Kanchipuram District, Tamil Nadu, India. Screening was carried out in Chunampet village for diabetes and diabetic eye diseases using a mobile telemedicine van.

A landmark study carried out between 2000 and 2001 by the Apollo Telemedicine Networking Foundation (6) was a major change and was instrumental in ISRO implementing telemedicine in India.

Teleophthalmology also address issues such as transportation, costs (7), concern over pupillary dilation, and adherence to recommended annual examination for chronic conditions like diabetic retinopathy and others.

Various studies indicate that telemedicine (8) increased the percentage of diabetic patients who underwent annual eye examination compared with traditional eye examination. Poor quality of images obtained from nonmydriatic cameras is the common cause for referral to the ophthalmologists.

In the various teleretinal screening programs (9) using nonmydriatic camera, diabetic retinopathy and other ocular diseases like age-related macular degeneration and others were diagnosed at remote areas.
The other eye care model in rural areas is Vision Centre. The model of Vision Centre (10) is envisaged by the Vision 2020-The Right to Sight, a global initiative of International Agency of Prevention of Blindness (IAPB, a global machinery working across the world for the prevention of avoidable blindness). IAPB has unveiled a four-tier pyramid model to provide eye care for the needy population where vision centers (11) are at the primary level. Aligning with this initiative, Government of India has plans to set up many vision centers across the country for providing basic eye care services on a permanent basis in villages. Each vision center will cover a population of about 45,000-50,000 patients.

All patients examined at the vision centers had teleconsultation with the ophthalmologist at the base eye hospital. Patients who required procedural intervention were asked to come to the hospital. These vision centers work closely with the community through community workers who create awareness about the eye problems in the community. In India, this model is implemented in the Aravind Eye Care System, Madurai, and at LV Prasad Eye Institute, Hyderabad.

In terms of diagnostic accuracy, real-time teleophthalmology (12) is considered equal to face-to-face consultation. Store-and-forward image transmission is a suitable alternative to overcome poor internet transmission speeds. 
A potential limitation of this study is that some rural areas had low internet connectivity and therefore did not support real-time videoconferencing. Although we had seen 1,05,827 patients, our teleconsultations were only 2,424 because of low internet bandwidth in remote villages.

\section{Conclusion}

Eye health promotion, prevention, diagnosis, and treatment of eye diseases in primary eye care and rehabilitation of those with irreversible blindness and low vision can be achieved using teleophthalmology. Having skilled local care providers (optometrists and ophthalmic technicians), trained in diagnostic instruments, imaging software, and hardware, has been identified as a success factor in implementing teleophthalmology (13). The Indian telemedicine establishments also need periodic evaluation to rationalize the main objective of the technology, that is, patient care, patient satisfaction (14), and patient opinion, all leading to patient empowerment in the community setting.

\section{Conflict of interest and funding}

The authors have not received any funding or benefits from industry to conduct this study.

\section{References}

1. Pascolini D, Mariotti SP. Global estimates of visual impairment: 2010. Br J Ophthalmol 2012; 96(5): 614-18. doi: 10.1136/ bjophthalmol-2011-300539

2. Das T, Pappuru RR. Telemedicine in diabetic retinopathy: access to rural India. Indian J Ophthalmol 2016; 64(1): 84-6. doi: 10.4103/0301-4738.178151

3. Ayatollahi H, Nourani A, Khodaveisi T, Aghaei H, Mohammadpour M. Teleophthalmology in practice: lessons learned from a pilot project. Open Med Informat J 2017; 11: 20. doi: $10.2174 / 1874431101711010020$
4. John S, Sengupta S, Reddy SJ, Prabhu P, Kirubanandan K, Badrinath SS. The Sankara Nethralaya mobile teleophthalmology model for comprehensive eye care delivery in rural India. Telemedicine e-Health 2012; 18(5): 382-7. doi: 10.1089/ tmj.2011.0190

5. Prathiba V, Rema M. Teleophthalmology: a model for eye care delivery in rural and underserved areas of India. Int J Family Med 2011; 2011: 683267. doi: 10.1155/2011/683267

6. Ganapathy K, Ravindra A. Telemedicine in India: the apollo story. Telemedicine e-Health 2009; 15(6): 576-85. doi: 10.1089/ tmj.2009.0066

7. Zimmer-Galler IE, Kimura AE, Gupta S. Diabetic retinopathy screening and the use of telemedicine. Curr Opin Ophthalmol 2015; 26(3): 167-72. doi: 10.1097/ICU.0000000000000142

8. Mansberger SL, Gleitsmann K, Gardiner S, Sheppler C, Demirel $\mathrm{S}$, Wooten $\mathrm{K}$, et al. Comparing the effectiveness of telemedicine and traditional surveillance in providing diabetic retinopathy screening examinations: a randomized controlled trial. Telemed J E Health 2013;19(2):942-8. doi: 10.1089/tmj.2012.0313

9. Maa AY, Patel S, Chasan JE, Delaune W, Lynch MG. Retrospective evaluation of a teleretinal screening program in detecting multiple nondiabetic eye diseases. Telemed e-Health 2017; 23(1): 41-8. doi: 10.1089/tmj.2016.0039

10. Qureshi B, Al-Rajhi A, Eckert K, Ravilla T, Khanna R, Mansur $\mathrm{R}$, et al. Best practice eye care models. Indian J Ophthalmol 2012; 60(5): 351. doi: 10.4103/0301-4738.100526

11. Kovai V, Rao GN, Holden B. Key factors determining success of primary eye care through vision centres in rural India: patients' perspectives. Indian J Ophthalmol 2007; 60(5): 487-91. doi: 10.4103/0301-4738.100558

12. Tan IJ, Dobson LP, Bartnik S, Muir J, Turner AW. Real-time teleophthalmology versus face-to-face consultation: a systematic review. J Telemed Telecare 2017; 23(7): 62. doi: 10.1177/ 1357633 X16660640

13. Caffery LJ, Taylor M, Gole G, Smith AC. Models of care in tele-ophthalmology: a scoping review. J Telemed Telecare 2019; 25(2): 106-22. doi: 10.1177/1357633X17742182

14. Paul PG, Raman R, Rani PK, Deshmukh H, Sharma T. Patient satisfaction levels during teleophthalmology consultation in rural South India. Telemed J e Health 2006; 12(5): 571-8. doi: 10.1089/tmj.2006.12.571 